\title{
Indian Mars craft prepares for orbit
}

\section{Mangalyaan aims to be Asia's first successful Martian mission.}

\section{BY SANJAY KUMAR}

T

ignesh Nair wanted to know the speed of the spacecraft; Mayyan Baatish asked why India was going to Mars at all, given the cost. For once, the 30-odd members of the Astronomy Club at the G. D. Goenka Public School in a Delhi suburb were discussing something home-grown: India's Mars spacecraft Mangalyaan, which is due to start orbiting the red planet on 24 September. If all goes according to plan, it will be the first successful Mars mission launched by an Asian nation - and a point of Indian pride.

Mangalyaan, known formally as the Mars Orbiter Mission, or MOM, was launched by the Indian Space Research Organisation (ISRO) last November. With 5 scientific instruments that collectively weigh just 15 kilograms, it is designed to image the planet and probe the composition of the surface and atmosphere, including testing for methane and measuring the ratio of deuterium to hydrogen.

Those are modest goals compared with, say, the much larger NASA orbiter MAVEN (Mars Atmosphere and Volatile Evolution), which is also en route to the red planet. Scheduled to arrive just three days ahead of MOM, it has eight instruments and would be the first spacecraft to examine questions such as how the solar wind has stripped away the Martian atmosphere.

As a result, the anticipation surrounding MOM comes not from the science, but from what a safe arrival would mean for India. "It will be a validation that Indian research and development has come of age," says Amitabha Ghosh, an Indian-born planetary scientist based in Washington DC. "India is still perceived as a place where work is outsourced, not because of superior science and engineering skills but because of a cost advantage."

ISRO has launched 35 satellites for countries including France, Germany, Canada, Israel and Singapore. Success for MOM could boost that commercial space industry, says Ajey Lele, a research fellow at the Institute for Defence Studies and Analysis, a think tank in New Delhi.

But trepidation still dogs the mission. MOM is about to enter a critical period: it has been in sleep mode for several months and must soon restart and then slow itself down, by firing its rockets for about 24 minutes, before it can enter Martian orbit. There is only one

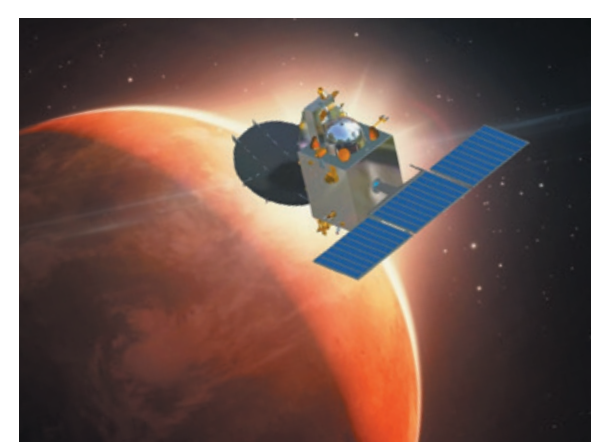

The Mars Orbiter Mission will hunt for methane.

opportunity for insertion, says A. S. Kiran Kumar, who runs ISRO's Space Applications Centre in Ahmedabad. "We are verifying everything daily and watching closely for any disturbance," he adds.

Ghosh worries that MOM's development was rushed, having taken just 15 months, according to ISRO. "A significant gestation period would have ensured proper engineering rigour and maximized the chances of success," he says.

Scientists also question the choice of rocket for the mission. MOM was launched using ISRO's low-power workhorse rocket, the Polar Satellite Launch Vehicle (PSLV), designed for putting satellites into low Earth orbit. That limited the weight of the payload - and, some suggest, MOM's scientific potential, although ISRO says that it miniaturized components to compensate. ISRO has been working for more than a decade and a half on the more powerful Geosynchronous Launch Vehicle, but the rocket has suffered a string of failures and did not manage to conduct a fully successful launch until January this year.

Whatever happens next, says Lele, India has learnt a lot from MOM. Using the lightweight PSLV at launch, for example, meant that the craft had to take a circuitous route to Mars, because it could not achieve the velocity needed to travel to the planet directly. "Being able to launch a robust spacecraft, overcoming minor glitches, sending it towards Mars innovatively, braving adverse space weather and radiation hazards while maintaining reliable communication for nearly ten months - it is already a significant achievement for India's first deep-space endeavour," says Lele. -

Additional reporting by Alexandra Witze. 